\title{
Replies to Reviewers
}

\section{Zhong et al, 'Dynamics of Fluorescent Imaging in Glob-Driven Breakup"}

We thank the reviewers for their comments and careful reading of the manuscript.

\section{Major Issues}

1. Page 4: in the characterization of the various numbers appearing in the nondimensional form of system (1)-(8), the Authors mention that the two Peclet numbers Pec and PeGamma are significantly greater than 1. It would be interesting if the Authors can: a. comment about the connection between PeGamma and the layer-like profile of Gamma in Fig. 1, second column from the left; b. provide details about the stabilization numerical approach (if any) they used to solve equations (2) and (3) which are strongly advection-dominated.

a. Since $\mathrm{Pe}_{\Gamma}$ is greater than one, we would expect that the advection is greater than diffusion for lipid. The steep layer from the initial condition is advected but $\Gamma$ also diffuses to the right. The rapid spread across the domain is predominantly by advection. But if we compute the solution for a longer time, the layer will be flattened more by diffusion. b. For the numerical methods, we first discretize the spatial derivative using Chebyshev spectral approximation and then use ode15s in Matlab to solve the resulting DAEs. Ode15s gives us stable results because there is still non-negligible diffusion present in $\Gamma$ and for the 4 th order equation for $h$. For clarification, we added a sentence to state the numerical methods that we use in the second paragraph of the second section. .

\section{Minor Issues}

1. page 2: line 4 from top: "Osmotic flow is assumed be proportional " $\rightarrow$ "Osmotic flow is assumed to be proportional"

" to" added

2. page 2 and 3: in the various figures the quantity alpha appears without ever being defined.

We add the definition of $a$ as required at the very end of section 3 in the following way:

" Here $a=1 / \sqrt{I_{0}}$ is the constant which normalizes $I$ to unity for $h=1$ at $t=0$." 